Annals of Pure and Applied Mathematics

Vol. 19, No. 1, 2019, 79-88

ISSN: 2279-087X (P), 2279-0888(online)

Published on 13 March 2019

Annals of

www.researchmathsci.org

DOI: http://dx.doi.org/10.22457/apam.601v19n1a10

Pure and Applied

Mathematics

\title{
A Type of the Cauchy-Euler Equations: Distinct Complex Roots
}

\section{Gumpon Sritanratana and Atiwath Chanram ${ }^{1}$}

Department of Mathematics Rajabhat Mahasarakham University, Mahasarakham 44000, Thailand. E-mail: sgumpon@gmail.com

${ }^{1}$ Corresponding author. E-mail: atiwath555@gmail.com

Received 2 February 2019; accepted 12 March 2019

Abstract. In this research, the authors give the family of all homogeneous Cauchy-Euler equations such that each equation has general solution depending on distinct complex numbers and their conjugates.

Keywords: Linear Differential Equations, Cauchy-Euler equations.

\section{AMS Mathematics Subject Classification (2010): 47E05}

\section{Introduction}

Consider a homogeneous Cauchy-Euler equation of order $n$ of the form

$$
a_{n} x^{n} \frac{d^{n} y}{d x^{n}}+a_{n-1} x^{n-1} \frac{d^{n-1} y}{d y^{n-1}}+\ldots+a_{1} x \frac{d y}{d x}+a_{0} y=0 .
$$

where $a_{0}, a_{1}, \ldots, a_{n}$ are real numbers with $a_{n} \neq 0$. Details for methods to find solutions of the equation (1.1) was explained in [2, 3, 4, 9]. Moreover, Sabuwala and Leon [7] studied the particular solution for the most general $n$-th order Euler differential equation when the non-homogeneity is a polynomial. They found a formula which can be used to compute the unknown coefficients in the form of the particular solution. It is well known that the general solution of (1.1) can be found from the characteristic equation

$$
\sum_{j=1}^{n} a_{j} \prod_{i=1}^{j}(m-i+1)+a_{0}=0
$$

of the linear ordinary differential equation with constant coefficients

$$
\left(\sum_{j=1}^{n} a_{j} \prod_{i=1}^{j}\left(\frac{d}{d t}-i+1\right)+a_{0}\right) y=0,
$$

where $t=\ln x$. In general, the general solution of any homogeneous Cauchy-Euler equations depends on zeros of the polynomial $\sum_{j=1}^{n} a_{j} \prod_{i=1}^{j}(m-i+1)+a_{0}$.

The aim of this paper is to give the family of all Cauchy-Euler equations (1.1) such that 


$$
\begin{gathered}
\text { Gumpon Sritanratana and Atiwath Chanram } \\
y=\sum_{j=1}^{k} x^{\alpha_{j}}\left(c_{2 j-1} \sin \left(\beta_{j} \ln x\right)+c_{2 j} \cos \left(\beta_{j} \ln x\right)\right)
\end{gathered}
$$

is the general solution of $(1.1)$ on $(0, \infty)$ where $\alpha_{j}$ and $\beta_{j}$ are real and imaginary parts of distinct $z_{j}$ with $\beta_{j} \neq 0$ for all $j=1, \ldots, k$.

\section{Preliminary}

In this section, we shall give the related basic notions that can be found in $[1,5,8]$.

Let $n \in \mathbb{N}, a_{0}, a_{1}, \ldots, a_{n} \in \mathbb{R}$ with $a_{n} \neq 0$. An ordinary differential equation of the form

$$
a_{n} \frac{d^{n} y}{d x^{n}}+a_{n-1} \frac{d^{n-1} y}{d y^{n-1}}+\cdots+a_{1} \frac{d y}{d x}+a_{0} y=0
$$

is said to be a homogeneous linear ordinary differential equation with constant coefficients. By a transformation $y=e^{m x}$, where $m$ is a suitable number, the equation (2.1) is transformed into the polynomial equation

$$
a_{n} m^{n}+a_{n-1} m^{n-1}+\ldots+a_{1} m+a_{0}=0,
$$

which is said to be the characteristic equation of (2.1).

A linear ordinary differential equation form

$$
a_{n} x^{n} \frac{d^{n} y}{d x^{n}}+a_{n-1} x^{n-1} \frac{d^{n-1} y}{d y^{n-1}}+\cdots+a_{1} x \frac{d y}{d x}+a_{0} y=0
$$

is called a homogeneous Cauchy-Euler equation.

Theorem 2.1. [5] Let $k$ be a positive integer, $n=2 k$ and $\alpha_{1}, \alpha_{2}, \ldots, \alpha_{k}, \beta_{1}, \beta_{2}, \ldots$, $\beta_{k}, a_{0}, a_{1}, \ldots, a_{2 k}$ be real numbers with $a_{n} \neq 0$. Then the distinct complex numbers $z_{1}=\alpha_{1}+\beta_{1} i, \quad z_{2}=\alpha_{2}+\beta_{2} i, \ldots, z_{k}=\alpha_{k}+\beta_{k} i, z_{k+1}=\overline{z_{1}}, \quad z_{k+2}=\overline{z_{2}}, \ldots, z_{2 k}=\overline{z_{k}}$ are distinct $2 k$ zeroes of the polynomial $\left(\sum_{j=1}^{n} a_{j} \prod_{i=1}^{j}\left(\frac{d}{d t}-i+1\right)+a_{0}\right) y=0$ if and only if

$$
y=\sum_{j=1}^{k} x^{\alpha_{j}}\left(c_{2 j-1} \sin \left(\beta_{j} \ln x\right)+c_{2 j} \cos \left(\beta_{j} \ln x\right)\right)
$$

is the general solution of homogeneous Cauchy-Euler equation (2.2) on an open interval $(0, \infty)$, where $c_{1}, c_{2}, \ldots, c_{2 k}$ are arbitrary constants.

Definition 2.1. [6] For each $j, k \in \mathbb{N}$ with $j \leq k$ we define

$$
\begin{gathered}
N_{k}:=\{1,2, \ldots, k\}, \\
P_{j, k}:=\left\{a_{1} a_{2} \cdots a_{j}: a_{1}, a_{2}, \ldots, a_{j} \in N_{k} \text { and } a_{1}<a_{2}<\cdots<a_{j}\right\}, \\
N_{j, k}:=\sum_{p \in P_{j, k}} p, \quad N_{0,0}:=1 \text { and } N_{0, k}:=1,
\end{gathered}
$$


A Type of the Cauchy-Euler Equations: Distinct Complex Roots

and for every integers $j, k$ with $k>j$ we define $N_{k, j}:=0$.

Lemma 2.1. [6] Let $n \in \mathbb{N}$ with $n \geq 2, m \in \mathbb{C}$ and $a_{1}, \ldots, a_{n} \in \mathbb{R}$ with $a_{n} \neq 0$. Then

$$
\sum_{j=1}^{n} a_{j} \prod_{i=1}^{j}(m-i+1)+a_{0}=a_{n} m^{n}+\sum_{j=1}^{n-1} m^{n-j} \sum_{i=0}^{j}(-1)^{i} N_{i, n+i-j-1} a_{n+i-j}+a_{0} .
$$

The following theorem is a direct consequence of Theorem 2.1 and Lemma 2.1.

Theorem 2.2. Let $k$ be a positive integer, $n=2 k$ and $\alpha_{1}, \alpha_{2}, \ldots, \alpha_{k}, \beta_{1}, \beta_{2}, \ldots, \beta_{k}$, $a_{0}, a_{1}, \ldots, a_{2 k}$ be real numbers with $a_{n} \neq 0$. Then the distinct complex numbers $z_{1}=\alpha_{1}+\beta_{1} i, \quad z_{2}=\alpha_{2}+\beta_{2} i, \ldots, z_{k}=\alpha_{k}+\beta_{k} i, \quad z_{k+1}=\overline{z_{1}}, \quad z_{k+2}=\overline{z_{2}}, \ldots, z_{2 k}=\overline{z_{k}}$ are distinct $2 k$ zeroes of the polynomial $a_{n} m^{n}+\sum_{j=1}^{n-1} m^{n-j} \sum_{i=0}^{j}(-1)^{i} N_{i, n+i-j-1} a_{n+i-j}+a_{0}$ if and only if (2.3) is the general solution of homogeneous Cauchy-Euler equation (2.2) on an open interval $(0, \infty)$, where $c_{1}, c_{2}, \ldots, c_{2 k}$ are arbitrary constants.

\section{Main theorems}

In this section, before proceeding to our main results, the following terminologies and concepts are required.

Definition 3.1. Let $n$ be a positive integer and $z_{1}, z_{2}, \ldots, z_{n}$ be distinct complex numbers. Then for every integer $j, k$ with $1 \leq j \leq k \leq n$ we define

$$
C_{k}=\left\{z_{1}, z_{2}, \ldots, z_{k}\right\}
$$

$S_{j, k}=\left\{\left\{a_{1}, a_{2}, \ldots, a_{j}\right\}: a_{1}, a_{2}, \ldots, a_{j} \in C_{k}\right.$ and $a_{1}, a_{2}, \ldots, a_{j}$ are distinct $\}$,

$$
C_{j, k}=\sum_{\left\{a_{1}, \ldots, a_{j}\right\} \in S_{j, k}} a_{1} a_{2} \cdots a_{j}, C_{0,0}=C_{0, j}=1,
$$

and $C_{k, j}=0$ for all $j<k \leq n$.

Form above definition, it is important to note that

$$
C_{k, k}=z_{1} z_{2} \cdots z_{k} \text { and } C_{k, k}=C_{k+1, k+1}
$$

for every positive integer $k$. Furthermore, we have the following applicable lemma.

Lemma 3.1. For every positive $n$. If $z_{1}, z_{2}, \ldots, z_{n}$ are distinct complex numbers and $C_{s}=\left\{z_{1}, z_{2}, \ldots, z_{s}\right\}$ for all positive integer $s$ with $s \leq n$, then for every positive integer $i, j$ with $i \leq j \leq n$,

$$
C_{i, j-1}+z_{j} C_{i-1, j-1}=C_{i, j}
$$




\section{Gumpon Sritanratana and Atiwath Chanram}

Proof: We shall proof by mathematical induction on $n$. For $n=2$, let $i, j$ be positive integers such that $i, j$ with $i \leq j \leq 2$ and $z_{1}, z_{2}$ are complex numbers and $C_{2}=\left\{z_{1}, z_{2}\right\}$. Then we have 3 cases: $i=j=1, i=1$ and $j=2$, and $i=j=2$. It follow that $C_{1,0}+z_{1} C_{1-1,1-1}=z_{1}=C_{1,1}, \quad C_{1,1}+z_{2} C_{1-1,2-1}=z_{1}+z_{2}=C_{1,2} \quad$ and $\quad C_{2,1}+z_{2} C_{2-1,2-1}=$ $z_{1} \cdot z_{2}=C_{2,2}$, this implies the lemma is true for $n=2$.

Next, we let $k$ be arbitrary positive integer with $k \geq 2$. Suppose that this lemma is true for $n=k$, that is for every distinct complex numbers $z_{1}, z_{2}, \ldots, z_{k}$ and $C_{s}=\left\{z_{1}\right.$, $\left.z_{2}, \ldots, z_{s}\right\}$ for all positive integer $s$ with $s \leq n$, for every positive integers $i, j$ with $i \leq j \leq k, C_{i, j-1}+z_{j} C_{i-1, j-1}=C_{i, j}$. Let $z_{1}, z_{2}, \ldots, z_{k+1}$ be distinct complex numbers and $C_{s}=\left\{z_{1}, z_{2}, \ldots, z_{s}\right\}$ for all positive integer $s$ with $s \leq k+1$. Let $i, j$ be positive integers with $i \leq j \leq k+1$. We shall proof that

$$
C_{i, j-1}+z_{j} C_{i-1, j-1}=C_{i, j} .
$$

Since $z_{1}, z_{2}, \ldots, z_{k}$ are complex numbers, from the inductive hypothesis, we obtain

$$
C_{i, j-1}+z_{j} C_{i-1, j-1}=C_{i, j} \text {. }
$$

for every positive integers $i, j$ with $i \leq j \leq k$, and so we only prove that

$$
C_{i, j-1}+z_{j} C_{i-1, j-1}=C_{i, j} \text {. }
$$

for all $i \leq k+1$. Let $i$ be a positive integer with $i \leq k+1$ and let

$$
R_{i, k+1}=\left\{\left\{a_{1}, a_{2}, \ldots, a_{i-1}, z_{k+1}\right\}: a_{1}, a_{2}, \ldots, a_{i-1} \in C_{k} \text { and } a_{1}, a_{2}, \ldots, a_{i-1}, z_{k+1} \text { are distinct }\right\} .
$$

We claim that $S_{i, k} \cap R_{i, k+1}=\varnothing$ and $S_{i, k} \cup R_{i, k+1}=S_{i, k+1}$. For $S_{i, k} \cap R_{i, k+1}=\varnothing$, suppose that $S_{i, k} \cap R_{i, k+1} \neq \emptyset$. Let $\left\{a_{1}, a_{2}, \ldots, a_{i}\right\} \in S_{i, k} \cap R_{i, k+1}$. Then $\left\{a_{1}, a_{2}, \ldots, a_{i}\right\} \in$ $S_{i, k}$ and $\left\{a_{1}, a_{2}, \ldots, a_{i}\right\} \in R_{i, k+1}$, and thus $a_{1}, a_{2}, \ldots, a_{i} \in C_{k}$ and $a_{1}, a_{2}, \ldots, a_{i}$ are distinct and there exists $j \in N_{i}$ such that $a_{j}=z_{k+1}$. Since $a_{s} \in C_{k}$ for every $s \in N_{i}$, we obtain $a_{s} \neq z_{k+1}$ which is impossible. Hence $S_{i, k} \cap R_{i, k+1}=\emptyset$.

Next, we shall prove that $S_{i, k} \cup R_{i, k+1}=S_{i, k+1}$. Let $\left\{a_{1}, a_{2}, \ldots, a_{i}\right\} \in S_{i, k} \cup R_{i, k+1}$. Then $\left\{a_{1}, a_{2}, \ldots, a_{i}\right\} \in S_{i, k}$ or $\left\{a_{1}, a_{2}, \ldots, a_{i}\right\} \in R_{i, k+1}$.

Case 1. Suppose that $\left\{a_{1}, a_{2}, \ldots, a_{i}\right\} \in S_{i, k}$. Then $a_{1}, a_{2}, \ldots, a_{i} \in C_{k}$ and $a_{1}, a_{2}, \ldots, a_{i}$ are distinct. Since $C_{k} \subseteq C_{k+1}, a_{1}, a_{2}, \ldots, a_{i} \in C_{k+1}$. It follows that $\left\{a_{1}, a_{2}, \ldots, a_{i}\right\} \in S_{i, k+1}$.

Case 2. Suppose that $\left\{a_{1}, a_{2}, \ldots, a_{i}\right\} \in R_{i, k+1}$. Then $a_{1}, a_{2}, \ldots, a_{j-1}, a_{j+1}, \ldots, a_{i} \in C_{k}$ are distinct element in $C_{k}$ and there exists $j \in N_{i}$ such that $a_{j}=z_{k+1}$ and thus $a_{1}, \ldots, a_{i}$ $\in C_{k+1}$. Hence $\left\{a_{1}, a_{2}, \ldots, a_{i}\right\} \in S_{i, k+1}$.

From both cases, we obtain $S_{i, k} \cup R_{i, k+1} \subseteq S_{i, k+1}$. 
A Type of the Cauchy-Euler Equations: Distinct Complex Roots

Now we let $\left\{a_{1}, a_{2}, \ldots, a_{i}\right\} \in S_{i, k+1}$. Then $a_{1}, a_{2}, \ldots, a_{i} \in C_{k+1}$ and $a_{1}, a_{2}, \ldots, a_{i}$ are distinct.

Case 1. Suppose that there exists $j \in N_{i}$ such that $a_{j}=z_{k+1}$. Since $a_{1}, a_{2}, \ldots, a_{i}$ are distinct complex numbers. Then $\left\{a_{1}, a_{2}, \ldots, a_{i}\right\} \in R_{i, k+1}$ and so $\left\{a_{1}, a_{2}, \ldots, a_{i}\right\} \in S_{i, k} \cup$ $R_{i, k+1}$.

Case 2. Suppose that $a_{j} \neq z_{k+1}$ for all $j \in N_{i}$. Since for every $j \in N_{i}, a_{i} \in C_{k+1}$, we obtain $a_{i} \in C_{k+1}$. Hence $\left\{a_{1}, a_{2}, \ldots, a_{i}\right\} \in S_{i, k}$ and thus $\left\{a_{1}, a_{2}, \ldots, a_{i}\right\} \in S_{i, k} \cup R_{i, k+1}$.

From both cases, we obtain $S_{i, k+1} \subseteq S_{i, k} \cup R_{i, k+1}$. It follows that $S_{i, k} \cup$ $R_{i, k+1}=S_{i, k+1}$. Hence

$$
\begin{aligned}
C_{i, k}+z_{k+1} C_{i-1, k} & =C_{i, k}+z_{k+1} \sum_{\left\{a_{1}, a_{2}, \ldots, a_{i-1}\right\} \in S_{i-1, k}} a_{1} a_{2} \cdots a_{i-1} \\
& =\sum_{\left\{a_{1}, a_{2}, \ldots, a_{i}\right\} \in S_{i, k}} a_{1} a_{2} \cdots a_{i}+\sum_{\left\{a_{1}, a_{2}, \ldots, a_{i-1}\right\} \in S_{i-1, k}} a_{1} a_{2} \cdots a_{i-1} z_{k+1} .
\end{aligned}
$$

Let $z_{k+1} S_{i-1, k}=\left\{\left\{a_{1}, a_{2}, \ldots, a_{i-1}, z_{k+1}\right\}:\left\{a_{1}, a_{2}, \ldots, a_{i-1}\right\} \in S_{i-1, k}\right\}$. Then

$$
\begin{aligned}
C_{i, k}+z_{k+1} C_{i-1, k} & =\sum_{\left\{a_{1}, a_{2}, \ldots, a_{i}\right\} \in S_{i, k}} a_{1} a_{2} \cdots a_{i}+\sum_{\left\{a_{1}, a_{2}, \ldots, a_{i-1}, z_{k+1}\right\} \in z_{k+1} S_{i-1, k}} a_{1} a_{2} \cdots a_{i-1} z_{k+1} \\
& =\sum_{\left\{a_{1}, a_{2}, \ldots, a_{i}\right\} a_{i, k}} a_{2} \cdots a_{i}+\sum_{\left\{a_{1}, a_{2}, \ldots, a_{i-1}, a_{i}\right\} \in z_{k+1} S_{i-1, k}} a_{1} a_{2} \cdots a_{i-1} a_{i} .
\end{aligned}
$$

Since $\left\{a_{1}, a_{2}, \ldots, a_{i-1}, a_{i}\right\} \in z_{k+1} S_{i-1, k}$

$$
\begin{aligned}
\Leftrightarrow & \text { there exists } j \in N_{i} \text { such that } a_{j}=z_{k+1} \text { and } \\
& a_{1}, a_{2}, \ldots, a_{j-1}, a_{j+1}, \ldots, a_{i-1}, a_{i} \in C_{k} \\
\Leftrightarrow & \left\{a_{1}, a_{2}, \ldots, a_{j-1}, z_{k+1}, a_{j+1}, \ldots, a_{i-1}, a_{i}\right\} \in R_{k+1} \\
\Leftrightarrow & \left\{a_{1}, a_{2}, \ldots, a_{i-1}, a_{i}\right\} \in R_{i, k+1},
\end{aligned}
$$

we obtain $z_{k+1} S_{i-1, k}=R_{k+1}$. Therefore

$$
C_{i, k}+z_{k+1} C_{i-1, k}=\sum_{\left\{a_{1}, a_{2}, \ldots, a_{i}\right\} \in S_{i, k}} a_{1} a_{2} \cdots a_{i}+\sum_{\left\{a_{1}, a_{2}, \ldots, a_{i}\right\} \in R_{k+1}} a_{1} a_{2} \cdots a_{i} .
$$

Since $S_{i, k} \cap R_{i, k+1}=\emptyset$ and $S_{i, k} \cup R_{i, k+1}=S_{i, k+1}$, we obtain

$$
C_{i, k}+z_{k+1} C_{i-1, k}=\sum_{\left\{a_{1}, a_{2}, \ldots, a_{i}\right\} \in S_{i, k} \cup R_{i, k+1}} a_{1} a_{2} \cdots a_{i}=\sum_{\left\{a_{1}, a_{2}, \ldots, a_{i}\right\} \in S_{i, k+1}} a_{1} a_{2} \cdots a_{i}=C_{i, k+1} .
$$

Hence $C_{i, k}+z_{k+1} C_{i-1, k}=C_{i, k+1}$.

By mathematical induction, this Lemma is true for all positive integer $n$.

Lemma 3.2. Let $n$ be a positive integer. If $z_{1}, z_{2}, \ldots, z_{n}$ are distinct complex numbers and $C_{n}=\left\{z_{1}, z_{2}, \ldots, z_{n}\right\}$, then 


\section{Gumpon Sritanratana and Atiwath Chanram}

$$
\prod_{i=1}^{n}\left(m-z_{i}\right)=\sum_{i=0}^{n-1}(-1)^{i} C_{i, n} m^{n-i}+(-1)^{n} C_{n, n} .
$$

Proof: We prove by mathematical induction. For $n=1$, let $z_{1}$ be a complex number.

Then since $C_{0,1}=1$ and $C_{1,1}=z_{1}$,

$$
\begin{aligned}
\prod_{i=1}^{1}\left(m-z_{i}\right) & =m-z_{1}=(-1)^{0} C_{0,1} m^{1-0}+(-1)^{1} C_{1,1}=\sum_{i=0}^{0}(-1)^{i} C_{i, 1} m^{1-i}+(-1)^{1} C_{1,1} \\
& =\sum_{i=0}^{n-1}(-1)^{i} C_{i, n} m^{n-i}+(-1)^{n} C_{n, n} .
\end{aligned}
$$

This implies that (3.2) is true for $n=1$.

Next, we let $k$ be a positive integer. Suppose that if $z_{1}, z_{2}, \ldots, z_{k}$ are distinct complex numbers and $C_{k}=\left\{z_{1}, z_{2}, \ldots, z_{k}\right\}$, then

$$
\prod_{i=1}^{k}\left(m-z_{i}\right)=\sum_{i=0}^{n-1}(-1)^{i} C_{i, n} m^{k-i}+(-1)^{k} C_{k, k} .
$$

Let $z_{1}, z_{2}, \ldots, z_{k}, z_{k+1}$ be distinct complex numbers and $C_{k+1}=\left\{z_{1}, z_{2}, \ldots, z_{k+1}\right\}$. Since

by the inductive hypothesis, we obtain

$$
\prod_{i=1}^{k+1}\left(m-z_{i}\right)=\left(m-z_{k+1}\right) \prod_{i=1}^{k}\left(m-z_{i}\right)
$$

and thus

$$
\prod_{i=1}^{k}\left(m-z_{i}\right)=\sum_{i=0}^{k-1}(-1)^{i} C_{i, k} m^{k-i}+(-1)^{k} C_{k, k},
$$

$$
\begin{aligned}
\prod_{i=1}^{k+1}\left(m-z_{i}\right)= & \left(m-z_{k+1}\right)\left(\sum_{i=0}^{k-1}(-1)^{i} C_{i, k} m^{k-i}+(-1)^{k} C_{k, k}\right) \\
= & \sum_{i=0}^{k-1}(-1)^{i} C_{i, k} m^{k+1-i}-\sum_{i=0}^{k-1}(-1)^{i} C_{i, k} m^{k-i} z_{k+1}+(-1)^{k} C_{k, k} m-(-1)^{k} z_{k+1} C_{k, k} \\
= & m^{k+1}+\sum_{i=1}^{k-1}(-1)^{i} C_{i, k} m^{k+1-i}-\sum_{i=0}^{k-2}(-1)^{i} C_{i, k} m^{k-i} z_{k+1} \\
& +(-1)^{k}\left(z_{k+1} C_{k-1, k}+C_{k, k}\right) m+(-1)^{k+1} C_{k+1, k+1} .
\end{aligned}
$$

By Lemma 3.1, we obtain $z_{k+1} C_{k-1, k}+C_{k, k}=C_{k, k+1}$ and therefore

$$
\begin{aligned}
\prod_{i=1}^{k+1}\left(m-z_{i}\right)= & m^{k+1}+\sum_{i=1}^{k-1}(-1)^{i} C_{i, k} m^{k+1-i}-\sum_{i=0}^{k-2}(-1)^{i} C_{i, k} m^{k-i} z_{k+1} \\
& +(-1)^{k} C_{k, k+1} m+(-1)^{k+1} C_{k+1, k+1} .
\end{aligned}
$$

Let $j=i-1$. Then $i=j+1$ and thus

$$
\prod_{i=1}^{k+1}\left(m-z_{i}\right)=m^{k+1}+\sum_{j=0}^{k-2}(-1)^{j+1} C_{j+1, k} m^{k-j}-\sum_{i=0}^{k-2}(-1)^{i} C_{i, k} m^{k-i} z_{k+1}
$$


A Type of the Cauchy-Euler Equations: Distinct Complex Roots

$$
\begin{aligned}
& +(-1)^{k} C_{k, k+1} m+(-1)^{k+1} C_{k+1, k+1} \\
= & m^{k+1}+\sum_{i=0}^{k-2}(-1)^{i+1} C_{i+1, k} m^{k-i}+\sum_{i=0}^{k-2}(-1)^{i+1} z_{k+1} C_{i, k} m^{k-i} \\
& +(-1)^{k} C_{k, k+1} m+(-1)^{k+1} C_{k+1, k+1} \\
= & m^{k+1}+\sum_{i=0}^{k-2}(-1)^{i+1} C_{i+1, k+1} m^{k-i}+(-1)^{k} C_{k, k+1} m+(-1)^{k+1} C_{k+1, k+1}
\end{aligned}
$$

by Lemma 3.1. Let $j=i+1$. Then $i=j-1$ and so

$$
\begin{aligned}
\prod_{i=1}^{k+1}\left(m-z_{i}\right) & =m^{k+1}+\sum_{j=1}^{k-1}(-1)^{j} C_{j, k+1} m^{k+1-j}+(-1)^{k} C_{k, k+1} m+(-1)^{k+1} C_{k+1, k+1} \\
& =m^{k+1}+\sum_{i=1}^{k-1}(-1)^{i} C_{j, k+1} m^{k+1-i}+(-1)^{k} C_{k, k+1} m+(-1)^{k+1} C_{k+1, k+1} \\
& =\sum_{i=0}^{k}(-1)^{i} C_{i, k+1} m^{k+1-i}+(-1)^{k+1} C_{k+1, k+1}
\end{aligned}
$$

The following corollary is a direct consequence of Lemma 3.2.

Corollary 3.1. Let $n$ be a positive integer. If $z_{1}, z_{2}, \ldots, z_{n}$ are distinct complex numbers and $C_{n}=\left\{z_{1}, z_{2}, \ldots, z_{n}\right\}$, then $z_{1}, z_{2}, \ldots, z_{n}$ are zeroes of the polynomial

$$
\sum_{i=1}^{n-1}(-1)^{i} C_{i, n} m^{n-i}+(-1)^{n} C_{n, n}
$$

Lemma 3.3. Let $n$ be a positive integer. If $z_{1}, z_{2}, \ldots, z_{n}$ are distinct complex numbers and $C_{n}=\left\{z_{1}, z_{2}, \ldots, z_{n}\right\}$, then

$$
a_{0}=(-1)^{n} C_{n, n}, \quad a_{n}=1 \text { and } a_{j}=(-1)^{n-j} C_{n-j, n}+\sum_{i=1}^{n-j}(-1)^{i+1} N_{i, i+j-1} a_{i+j}
$$

for every $j=1,2, \ldots, n-1$ if and only if

$$
a_{n} m^{n}+\sum_{j=1}^{n-1} m^{n-j} \sum_{i=0}^{j}(-1)^{i} N_{i, n+i-j-1} a_{n+i-j}+a_{0}=\sum_{i=0}^{n-1}(-1)^{i} C_{i, n} m^{n-i}+(-1)^{n} C_{n, n} .
$$

Proof: Suppose that (3.4) is true for all $j=1,2, \ldots, n-1$. Then for any $j=1,2, \ldots, n-1$,

$$
a_{j}+\sum_{i=1}^{n-j}(-1)^{i} N_{i, i+j-1} a_{i+j}=(-1)^{n-j} C_{i, n}
$$

that is for every $s=1,2, \ldots, n-1$,

$$
a_{s}+\sum_{i=1}^{n-s}(-1)^{i} N_{i, i+s-1} a_{i+s}=(-1)^{n-s} C_{i, n} .
$$

Let $s=n-j$. Then $j=n-s$. and thus for every $j=1,2, \ldots, n-1$, 


\section{Gumpon Sritanratana and Atiwath Chanram}

$$
a_{n-j}+\sum_{i=1}^{j}(-1)^{i} N_{i, n+i-j-1} a_{n+i-j}=(-1)^{j} C_{i, n} .
$$

Since $N_{0, n-j-1}=1$,

$$
(-1)^{0} N_{0, n-j-1} a_{n-j}+\sum_{i=1}^{j}(-1)^{i} N_{i, n+i-j-1} a_{n+i-j}=(-1)^{j} C_{i, n}
$$

and thus

$$
\sum_{i=0}^{j}(-1)^{i} N_{i, n+i-j-1} a_{n+i-j}=(-1)^{j} C_{i, n},
$$

for every $j=1,2, \ldots, n-1$. Multiplying both sides of this equation by $m^{n-j}$, we obtain

$$
m^{n-j} \sum_{i=0}^{j}(-1)^{i} N_{i, n+i-j-1} a_{n+i-j}=(-1)^{j} C_{i, n} m^{n-j} .
$$

Adding this $n-1$ equations, we have

$$
\sum_{j=1}^{n-1} m^{n-j} \sum_{i=0}^{j}(-1)^{i} N_{i, n+i-j-1} a_{n+i-j}=\sum_{j=1}^{n-1}(-1)^{j} C_{i, n} m^{n-j} .
$$

That is

$$
a_{n} m^{n}+\sum_{j=1}^{n-1} m^{n-j} \sum_{i=0}^{j}(-1)^{i} N_{i, n+i-j-1} a_{n+i-j}+a_{0}=\sum_{i=0}^{n-1}(-1)^{i} C_{i, n} m^{n-i}+(-1)^{n} C_{n, n} .
$$

Conversely, assume that the equation (3.5) is true. Since $1, m, m^{2}, \ldots, m^{n}$ are linearly independent, by undetermined coefficients, we obtain

$$
a_{n}=1, \quad a_{0}=(-1)^{n} C_{n, n}
$$

and for every $j=1,2, \ldots, n-1$,

that is

$$
\sum_{i=0}^{j}(-1)^{i} N_{i, n+i-j-1} a_{n+i-j}=\sum_{j=1}^{n-1}(-1)^{j} C_{j, n},
$$

$$
a_{n-j}+\sum_{i=1}^{j}(-1)^{i} N_{i, n+i-j-1} a_{n+i-j}=(-1)^{j} C_{j, n},
$$

Let $s=n-j$. Then for every $s=1,2, \ldots, n-1$,

$$
a_{s}+\sum_{i=1}^{n-s}(-1)^{i} N_{i, i+s-1} a_{i+s}=(-1)^{n-s} C_{n-s, n}
$$

and thus for every $j=1,2, \ldots, n-1$,

$$
a_{j}=(-1)^{n-j} C_{n-j, n}+\sum_{i=1}^{n-j}(-1)^{i+1} N_{i, i+j-1} a_{i+j} .
$$

The proof is complete. 
A Type of the Cauchy-Euler Equations: Distinct Complex Roots

Corollary 3.2. Let $k$ be a positive integer and $\alpha_{1}, \alpha_{2}, \ldots, \alpha_{k}, \beta_{1}, \beta_{2}, \ldots, \beta_{k}, a_{0}, a_{1}, \ldots, a_{2 k}$ be real numbers with $a_{n} \neq 0$. If $z_{1}, z_{2}, \ldots, z_{2 k}$ are distinct complex numbers and $C_{2 k}=$ $\left\{z_{1}, z_{2}, \ldots, z_{2 k}\right\}$ such that $z_{1}=\alpha_{1}+\beta_{1} i, \quad z_{2}=\alpha_{2}+\beta_{2} i, \ldots, z_{k}=\alpha_{k}+\beta_{k} i, \quad z_{k+1}=\overline{z_{1}}$, $z_{k+2}=\overline{z_{2}}, \ldots, z_{2 k}=\overline{z_{k}}$. Then

$$
a_{0}=(-1)^{2 k} C_{2 k, 2 k}, \quad a_{2 k}=1 \text { and } a_{j}=(-1)^{2 k-j} C_{2 k-j, 2 k}+\sum_{i=1}^{2 k-j}(-1)^{i+1} N_{i, i+j-1} a_{i+j}
$$

for every $j=1,2, \ldots, n-1$ if and only if $z_{1}, z_{2}, \ldots, z_{2 k}$ are the zeroes of polynomial

$$
a_{2 k} m^{2 k}+\sum_{j=1}^{2 k-1} m^{2 k-j} \sum_{i=0}^{j}(-1)^{i} N_{i, 2 k+i-j-1} a_{2 k+i-j}+a_{0} .
$$

Proof: By Lemma 3.3 with $n=2 k$, the formulas (3.6) is true for every $j=1,2, \ldots, 2 k-1$ if and only if

$$
a_{2 k} m^{2 k}+\sum_{j=1}^{2 k-1} m^{2 k-j} \sum_{i=0}^{j}(-1)^{i} N_{i, 2 k+i-j-1} a_{2 k+i-j}+a_{0}=\sum_{i=0}^{2 k-1}(-1)^{i} C_{i, 2 k} m^{2 k-i}+(-1)^{2 k} C_{2 k, 2 k} .
$$

By Corollary 3.1 with $n=2 k$, we obtain

$$
\prod_{i=1}^{2 k}\left(m-z_{i}\right)=\sum_{i=0}^{2 k-1}(-1)^{i} C_{i, 2 k} m^{2 k-i}+(-1)^{2 k} C_{2 k, 2 k} .
$$

and thus (3.6) is true for every $j=1,2, \ldots, n-1$ if and only if

$$
\prod_{i=1}^{n}\left(m-z_{i}\right)=a_{n} m^{n}+\sum_{j=1}^{n-1} m^{n-j} \sum_{i=0}^{j}(-1)^{i} N_{i, n+i-j-1} a_{n+i-j}+a_{0} .
$$

Thus (3.6) holds for every $j=1,2, \ldots, n-1$, if and only if $z_{1}, z_{2}, \ldots, z_{n}$ are zeroes of the polynomial (3.7).

The following main theorem is a direct consequence of Corollary 3.2 and Theorem 2.2 with $n=2 k$.

Theorem 3.1. Let $k$ be a positive integer and $\alpha_{1}, \alpha_{2}, \ldots, \alpha_{k}, \beta_{1}, \beta_{2}, \ldots, \beta_{k}, a_{0}, a_{1}, \ldots, a_{2 k}$ be real numbers with $a_{2 k} \neq 0$. If $z_{1}, z_{2}, \ldots, z_{2 k}$ are distinct complex numbers and $C_{2 k}=\left\{z_{1}, z_{2}, \ldots, z_{2 k}\right\} \quad$ such that $z_{1}=\alpha_{1}+\beta_{1} i, \quad z_{2}=\alpha_{2}+\beta_{2} i, \ldots, z_{k}=\alpha_{k}+\beta_{k} i$, $z_{k+1}=\overline{z_{1}}, z_{k+2}=\overline{z_{2}}, \ldots, z_{2 k}=\overline{z_{k}}$. Then (3.6) is true for every $j=1,2, \ldots, 2 k-1$ if and only if

$$
y=\sum_{j=1}^{k} x^{\alpha_{j}}\left(c_{2 j-1} \sin \left(\beta_{j} \ln x\right)+c_{2 j} \cos \left(\beta_{j} \ln x\right)\right)
$$

is the general solution of homogeneous Cauchy-Euler equation

$$
a_{2 k} x^{2 k} \frac{d^{2 k} y}{d x^{2 k}}+a_{2 k-1} x^{2 k-1} \frac{d^{2 k-1} y}{d x^{2 k-1}}+\cdots+a_{1} x \frac{d y}{d x}+a_{0} y=0
$$




\section{Gumpon Sritanratana and Atiwath Chanram}

on an open interval $(0, \infty)$, where $c_{1}, c_{2}, \ldots, c_{2 k}$ are arbitrary constants.

The application of Theorem 3.1 is to establish a Cauchy-Euler equation of order $n$ together with its general solution on $(0, \infty)$, of the form (3.8).

\section{Conclusion}

We give every Cauchy-Euler differential equation from its general solution that depends only on a given finite numbers of distinct complex numbers. In the future, we will devote our attention to the family of all Cauchy-Euler differential equations that have general solutions depending only on a complex number.

Acknowledgements. The authors are thankful to the reviewers for valuable comments and suggestions on the manuscript and thank the Faculty of Science and Technology, Rajabhat Mahasarakham University, Mahasarakham, Thailand for financial support.

\section{REFERENCES}

1. S.Ahmad and A.Ambrosetti, A Textbook on ordinary differential equations, Springer International Publishing Switzerland, (2015).

2. W.E.Boyce and R.C.DiPrima, Elementary differential equations and boundary value problems, Seventh edition, John Wiley \& Sons, Inc., New York-London-Sydney, (2001).

3. E.A.Coddington, An introduction to ordinary differential equations, Prentice-Hall Mathematics Series Prentice-Hall, Inc., Englewood Cliffs, N.J., (1961).

4. E.A.Coddington, N.Levinson, Theory of ordinary differential equations, McGrawHill Book Company, Inc., New York-Toronto-London, (1995).

5. S.W.Goode and S.A.Annin, Differential equations and linear algebra, fourth edition, Pearson Education, Inc., (2015).

6. W.Jisabuy and G.Sritanratana, A type of the Cauchy-Euler Equations: A unique real root, Annals of Pure and Applied Mathematics, 18 (1) (2018) 27-35.

7. A.H.Sabuwala and D.De Leon, Particular solution to the Euler-Cauchy equation with polynomial non-homogeneities, Discrete Contin. Dyn. Syst., Dynamical Systems, Differential Equations and Applications, 8th AIMS Conference. Suppl. 2 (2011), $1271-1278$.

8. D.A.Sanchez, Ordinary differential equations and stability theory an introduction, Dover Publications Inc., (1979).

9. D.Zill and W.Wright, Differential equations and boundary value problems, eighth edition, Brooks/Cole, Boston, (2013). 